\title{
Lowbush blueberries, Vaccinium angustifolium, modulate the functional potential of nutrient utilization and DNA maintenance mechanisms in the rat proximal colon microbiota
}

\author{
Alison Lacombe ${ }^{a}$, Robert W. Li ${ }^{\text {b1 }}$, Dorothy Klimis-Zacas ${ }^{a}$, Aleksandra S. Kristo ${ }^{a}$, \\ Shravani Tadepalli ${ }^{\mathrm{a}}$, Emily Krauss ${ }^{\mathrm{a}}$, Ryan Young ${ }^{\mathrm{c}}$, and Vivian C.H. Wu*a \\ ${ }^{a}$ Department of Food Science and Human Nutrition, The University of Maine, 5735 Hitchner

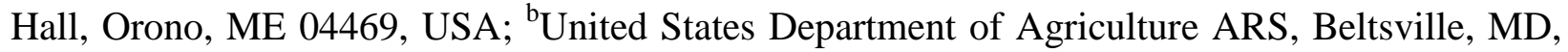 \\ USA ; ${ }^{c}$ Department of Molecular and Biomedical Sciences, The University of Maine, Orono, \\ ME, 04469, USA
}

*Corresponding Author: Vivian C.H. Wu, Department of Molecular and Biomedical Sciences, The University of Maine, Orono, ME, 04469, USA

Submission date: May 15, 2012, Acceptance date: June 14, 2012; Publication date: June 18, 2012

\begin{abstract}
:
Background: The core gut microbiota encodes for a metabolic capacity that often surpasses the metabolic potential of its host. Lowbush wild blueberries (LWB) are a rich source of bioactive compounds, and the gut microbiota is an important modulator of their activity. The objective of this research is to study the effect of a diet enriched with LWB on the gastrointestinal microbiota's metabolic potential.
\end{abstract}

Methods: Nine three-week-old male Sprague Dawley rats were randomly assigned to two groups. The control group $(N=4)$ was placed on the AIN93 diet, and the treatment group $(N=5)$ was fed the same diet with $8 \%(\mathrm{w} / \mathrm{w})$ LWB powder substituting for dextrose. The animals consumed the diets for six weeks, after which they were sacrificed. Functional profiles and metabolic potential of the colon microbiota in response to diet were analyzed using deep whole genome sequencing (WGS).

Results: Proteins predicted from WGS DNA sequences were assigned to 3,746 COG, 5,577 KEGG, and 4,474 Pfam families. Statistical comparisons of the protein-coding genes revealed significant changes in 25 Gene Ontology (GO), 186 KEGG, and 20 Pfam protein families representing $2.1 \%, 3.3 \%$, and $0.02 \%$ of all hits identified, respectively. Overall, the combined inquires into these databases represented an emphasis on membrane trafficking, DNA regulation, modification and repair, and nutrient metabolism. Protein families that significantly increased by the enrichment of LWBs were involved with amino acid metabolism, 2,4-dienoyl-CoA reductase, metal ion binding, glutamate synthase, REDOX homeostasis, and aryl transferases. In 
contrast, protein families involved with integrase/recombinase, reverse transcriptase, and transposon/transposase were at a lower abundance.

Conclusions: The results from this study reflect the potential impact that LWBs have on the functional repertoire of bacterial communities in the proximal colon. The results demonstrate a higher potential for the metabolism of amino acids and a lower potential for horizontal integration of plasmids and lower phage/transposon activity in the LWB diet, suggesting that the genome at the time of sampling was more stable. This study helps unravel diverse mechanisms of microbial adaptation to LWBs in the diet of mammals and can provide guidance in optimizing functional diets.

\section{INTRODUCTION:}

The intestinal microbiome is closely involved in the host's health, modulating the extraction of energy from otherwise indigestible compounds, the stimulation of the gut immune system, the regulation of cell proliferation, the synthesis of vitamins $\mathrm{K}$ and $\mathrm{B}$, and the enhancement of pathogen resistance [1]. In addition, the disequilibrium of the intestinal microbiome is considered a risk factor contributing to diseases ranging from allergies, bowel inflammation, cardiovascular disease, diabetes, and obesity [1]. The microbial genome reflects catabolic, physiological, and biosynthetic capabilities of microorganisms, and its ability to adapt to environmental change. Recent developments in molecular biology allow for the simultaneous analysis of genes and have revealed insights into the molecular basis of the native gut microbiota in response to dietary changes [2].

The lowbush wild blueberry (LWB), Vaccinium angustifolium, exhibits the highest antioxidant and cellular protective effects [3]. Lowbush wild blueberries are high in polyphenols, fibers, sitosterol, manganese, vitamins $\mathrm{B}_{6}, \mathrm{C}$, and $\mathrm{K}$, and represent the highest source of anthocyanins in the American diet [4]. Although LWBs were found to benefit several aspects of human health, little is known about how their bio-protective effects become available to their host. The oxygen-scavenging capabilities of polyphenols have been shown in vitro to protect host tissue, while having inhibitory effects against pathogenic microorganisms and cancer cells [5, 6]. Many in vitro studies have demonstrated the potential benefits of consuming LWBs, but few extend their results to in vivo situations [7]. For example, the absorption of polyphenols is highly varied based upon the glycosylation and the degree polymerization and the ability of the mammalian intestinal $\beta$-glucosidase. In humans, the intestinal absorption of dietary polyphenols is often slow and largely incomplete, and up to $85 \%$ of LWB anthocyanins enter the colon intact [8]. In the colon, polyphenols are processed through a complex and diverse bioreactor consisting of $10^{9}-10^{12}$ microorganisms. The microbes that reside in the colon are capable of catabolizing polyphenols through demethylation, dehydroxlyations, and ring cleavages [9]. These reactions may have impacts of the biological activity of these compounds.

Lowbush wild blueberries may have an impact on cellular mechanics and central metabolism of the core microbiota [9-11]. There are several metabolic pathways proposed for the catabolism of phenolic acids, anthocyanins, and proanthocyanidins by the intestinal microbiota $[9,12]$. Catabolic action by host intestinal enzymes or native gut flora contributes to the 
bioavailability of these nutrients [12]. Different metabolic pathways for the digestion of polyphenols could be attributed to variations in the microbiota composition, and different pathways could coexist, depending on the catabolic capacity of the microbiota [13]. The enzymes of these pathways, coded for by genes, can be specific for certain growth substrates, and are generally induced by the corresponding substrate. Until recently, research in this area has been limited because the technology lacks the resolution to conduct multi-species inquires. Advances in next generation DNA-sequencing technology has dramatically changed the way scientists investigate the microbial communities that populate the gut [14]. Metagenomic studies can resolve a global snapshot of an entire community by describing the functional genomics of microbial communities and their physiological phenotypes [14]. This technology has enormous potential and can be utilized to investigate the microbial function in response to dietary alterations.

There is a lack of knowledge with respect to how blueberries affect the function and cellular mechanics of the gut microbes and how this interaction could potentially impart health benefits to the host. The objectives of this research were to i) characterize the function of the microbial community of the Sprague Dawley (SD) rats using metagenomic techniques and ii) utilize this model to detect functional changes of the metabolic pathways, cellular function, and nutrient utilization in a LWB enriched diet. By indentifying microbial functional genes in the Cluster of Orthologous Groups (COG), Kyoto Encyclopedia of Genes and Genomes (KEGG), Gene Ontology (GO), and Protein Family Database (Pfam) databases, metabolic difference in gut microbiota may be detected. Investigating functional changes in gut microbiota in response to LWB enrichment may provide insights into how LWB impart health benefits to their host.

\section{MATERIALS AND METHODS:}

\subsection{Animal diet, feeding, and tissue sampling}

Nine male SD rats, three-weeks old and approximately $90 \mathrm{~g}$, were purchased from Charles River Laboratories (Wilmington, MA). They were randomly divided into two groups: the control group $(N=4)$ was fed a control diet (AIN93) [15], and the experimental group $(N=5)$ was fed a LWB enriched diet (AIN93 + 8\% w/w LWB powder substituting for dextrose). Tap water and diet were provided ad libitum, and animals consumed $20 \pm 4 \mathrm{~g}$ of feed per day, an amount equivalent to $24.0 \pm 5.2 \mathrm{mg}$ of anthocyanins per day [16]. After the feeding period of six weeks, animals were sacrificed under $95 \% \mathrm{CO}_{2} / 5 \% \mathrm{O}_{2}$ inhalation for three minutes. Samples for metagenomic analysis were taken from the proximal colon, approximately one inch downstream of the cecum. The colon contents $(0.37 \pm 0.13 \mathrm{~g})$ were collected by elevating one end of the large intestine and pushing contents into a sterile sample container. The samples were then snap-frozen in liquid nitrogen and stored at $-80^{\circ} \mathrm{C}$. The study was carried out in strict accordance with the recommendations in the Guide for the Care and Use of Laboratory Animals of the National Institutes of Health. All animal procedures were approved by the Institutional Animal Care and Use Committees of the University of Maine (Protocol \#A2011-01-03).

\subsection{Metagenomic DNA extraction and sequencing}

Metagenomic DNA extraction was executed using a QIAamp DNA stool kit (Qiagen, Valenica, CA) with modifications to the protocol described by $\mathrm{Li}$ et al., 2011. Lysis incubation at $95^{\circ} \mathrm{C}$ for 
six minutes was used to replace the $70^{\circ} \mathrm{C}$ lysis recommended in the standard protocol. DNA integrity was verified using a Bioanalyzer 2100 (Agilent, Palo Alto, CA). Metagenomic DNA concentration was quantified using a QuantiFlour flourometer (Promega, Madison, WI). Approximately $1.0 \mu \mathrm{g}$ of high-quality DNA was processed using an Illumina TruSeq DNA sample prep kit following manufacturer's instruction (Illumina, San Diego, CA). Final individual libraries were validated and pooled based on their respective 6-bp adaptors then sequenced at 100bp/sequence read using an Illumina HiSeq 2000 sequencer. Raw sequence reads generated for this study were previously described [17]. All Illumina raw sequences were deposited into MG-RAST databases (MG-RAST Accession \# from 4470921.3 to 4471270.3) (http://metagenomics.anl.gov).

\subsection{Data analysis and statistics}

Sequence reads from the whole genome shotgun (WGS) approach were first trimmed using SolexaQA, a Perl-based software package calculating quality statistics from FASTQ files generated by Illumina sequencers. Before analysis, quality control filters were applied to WGS raw reads. Mammalian contaminants were removed using FR-HIT, and redundant reads were identified and removed using CD-HIT [17]. The remainder of WGS sequences was de novo assembled using SOAPdenovo software package (http://soap.genomics.org.cn/soapdenovo.html) Open reading frames (ORFs) were predicted from contigs $\geq 200 \mathrm{bp}$ using FragGeneScan (v1.14). Functional annotation was performed according to the COG, KEGG, and Pfam (v24.0) databases using RPS-BLAST (v2.2.15), BLASTP (v2.2.15), and HMMSCAN (v3.0) software, respectively.

\section{RESULTS:}

\subsection{The protein repertoire of the rat proximal colon microbiota}

The functional and metabolic potentials of the rat proximal colon microbiota of both diets were evaluated using assembled WGS sequence reads. A total of 3,746 COG, 5,577 KEGG, 1,164 GO, and 4,474 Pfam protein families were identified in the rat proximal colon microbiota. The most abundant COG protein classes identified in the control diet pertained to carbohydrates (Class G), amino acids (Class E), and DNA replication, recombination, and repair (Class L) (Figure 1).

The highest percent abundant COG protein families in the microbiota of control animals included $\mathrm{Na}^{+}$driven multidrug pump $(0.74 \%$ control; $0.81 \%$ blueberry), response regulators to helix-turn-helix $(0.70 \%$ control; $0.67 \%$ blueberry), signal transduction histidine kinase $(0.68 \%$ control; $0.61 \%$ blueberry), DNA-directed RNA polymerase specialized sigma subunit $(0.33 \%$ control; $0.53 \%$ blueberry), and ATP Binding Cassette (ABC)-type multidrug transport system ( $0.47 \%$ control; $0.47 \%$ blueberry). Predicted ORFs were also annotated against the KEGG and Pfam databases for both diets and demonstrated similar results with respect to COG proteins identified. The most abundant KEGG protein families in the rats were RNA polymerase sigma 70 factor $(0.60 \%$ control; $0.48 \%$ blueberry), site-specific DNA recombinase $(0.56 \%$ control; $0.48 \%$ blueberry), $\mathrm{ABC}$ subfamily $\mathrm{B}$ ( $0.48 \%$ control; $0.60 \%$ blueberry), iron complex outer membrane receptor $(0.44 \%$ control; 0.38 blueberry), integrase/recombinase XerD $(0.44 \%$ control; $0.38 \%$ blueberry), and $\beta$-galactosidase ( $0.40 \%$ control; $0.41 \%$ blueberry). 


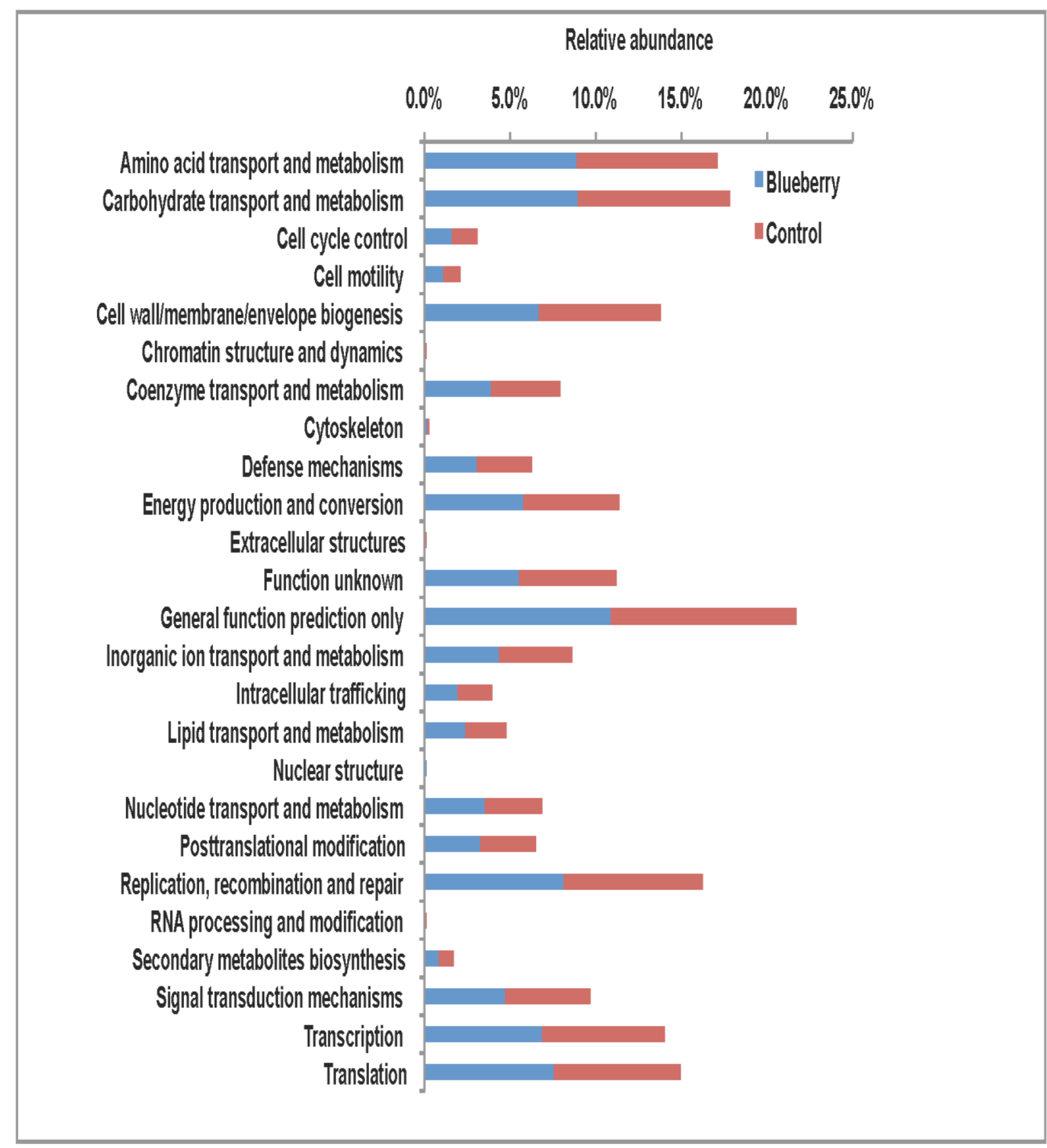

Figure 1: COG function classes identified in the rat proximal colon microbiota.

Pfam protein families are listed in Table 1 and include $\mathrm{ABC}$ transporters, response regulator receiver domain, histidine kinase, multidrug extrusion protein (MatE), and helix-turn-helix as being highly represented. The GO terms in high abundance involved ATP binding (5.8\% control; $5.7 \%$ blueberry), membrane function ( $4.4 \%$ control; $4.3 \%$ blueberry), and DNA binding activity (3.6\% control; $3.5 \%$ blueberry). Overall, the combined inquires into the COG, KEGG, Pfam, and GO databases represent the gut microbiota emphasis on membrane trafficking, DNA regulation, modification and repair, and nutrient metabolism. 
Table 1. The most abundant Protein Family Database (Pfam) protein families detected in the proximal colon microbiota of rats. No significant difference was detected between the two groups.

\begin{tabular}{llll}
\hline & & & Blueberry \\
Pfam_ID & Description & Control (N=4) & (N=5) \\
\hline PF00005 & ABC transporter & $1.42 \pm 0.08$ & $1.39 \pm 0.03$ \\
PF00072 & Response regulator receiver domain & $0.88 \pm 0.06$ & $0.84 \pm 0.03$ \\
PF02518 & Histidine kinase-, DNA gyrase B-, and HSP90-like ATPase & $0.77 \pm 0.03$ & $0.70 \pm 0.02$ \\
PF01554 & MatE & $0.66 \pm 0.03$ & $0.72 \pm 0.01$ \\
PF01381 & Helix-turn-helix & $0.61 \pm 0.03$ & $0.60 \pm 0.05$ \\
& Binding-protein-dependent transport $\quad$ (inner $\quad$ membrane & & \\
PF00528 & component) & $0.61 \pm 0.12$ & $0.59 \pm 0.02$ \\
PF00589 & Phage integrase family & $0.54 \pm 0.03$ & $0.52 \pm 0.04$ \\
PF00165 & Bacterial regulatory helix-turn-helix proteins, AraC family & $0.49 \pm 0.04$ & $0.37 \pm 0.03$ \\
PF00593 & TonB dependent receptor & $0.47 \pm 0.09$ & $0.41 \pm 0.05$ \\
& ATPase family associated with various cellular activities & & \\
PF00004 & (AAA) & $0.47 \pm 0.00$ & $0.47 \pm 0.01$ \\
PF00535 & Glycosyl transferase family 2 & $0.45 \pm 0.05$ & $0.38 \pm 0.02$ \\
PF07715 & TonB-dependent Receptor Plug Domain & $0.45 \pm 0.08$ & $0.41 \pm 0.04$ \\
PF00512 & His Kinase A (phosphoacceptor) domain & $0.44 \pm 0.01$ & $0.40 \pm 0.02$ \\
PF04542 & Sigma-70, region 2 & $0.39 \pm 0.03$ & $0.40 \pm 0.02$ \\
PF08281 & Sigma-70, region 4 & $0.39 \pm 0.03$ & $0.40 \pm 0.02$ \\
PF00009 & Elongation factor Tu GTP binding domain & $0.39 \pm 0.02$ & $0.39 \pm 0.01$ \\
PF07690 & Major Facilitator Superfamily & $0.39 \pm 0.04$ & $0.31 \pm 0.01$ \\
PF00155 & Aminotransferase class I and II & $0.39 \pm 0.00$ & $0.39 \pm 0.00$ \\
PF01370 & NAD dependent epimerase/dehydratase family & $0.37 \pm 0.02$ & $0.31 \pm 0.01$ \\
PF00873 & AcrB/AcrD/AcrF family & $0.35 \pm 0.01$ & $0.30 \pm 0.02$ \\
\hline
\end{tabular}

\section{Lowbush wild blueberry diet impacts the functional potential of the microbiota in the rat proximal colon}

Our results demonstrated that the LWB was able to induce a significant impact $(P<0.05)$ on 25 GO terms, $186 \mathrm{KEGG}$, and 20 Pfam protein families, which represent $2.1 \%, 3.3 \%, 0.02 \%$ of all hits in each category identified, respectively. Significant increases in COG functional class induced by the blueberry-enriched diet were observed in amino acid metabolism (Class E). The LWB diets observe a higher abundance in Pfam proteins involved with the S-layer cell envelope proteins, solenoid proteins involved in signal transduction (Sel1), and collagen binding protein (Cna). GO terms associated with histidine and cellular amino acid biosynthetic processes and amino acid metabolic processes were significantly more abundant in the proximal colon microbiota of rats fed a LWB-enriched diet. The GO platform detected overall cellular metabolic processes at a $0.03 \%$ lower abundance in the LWB diet (Table 2). The KEGG database indentified a $0.07 \%$ increase in CDRs and OFRs for glutamate synthase (Figure 2), and a decrease in aspartate aminotransferase $(0.02 \%)$ and histidine kinase $(0.02 \%)$ in the LWB diet. Additionally, the LWB diet increased Pfam protein families associated with amino acid metabolism, such as, aminotransferase, peptidase family M23, and branched chain amino acid 
transport systems by $0.03 \%, 0.01 \%$, and $0.09 \%$ abundance, respectively (Table 3 ). The overall increases in the percent abundance of genes associated with amino acids in the LWB is reflected in the KEGG, COG, and Pfam demonstrates significant impact on this aspect of metabolism.

Table 2. Gene Ontology (GO) significantly impacted by a lowbush wild blueberry enriched diet. * $P$ value was calculated based on unpaired t-test. The number denotes the percentage of hits annotated to a given functional class \pm standard error of the mean.

\begin{tabular}{|c|c|c|c|c|}
\hline GO_id & Description & Control $(N=4)$ & Blueberry $(N=5)$ & $\begin{array}{l}* P \\
\text { value }\end{array}$ \\
\hline GO:0055085 & transmembrane transport & $1.498 \pm 2.68 \times 10^{-4}$ & $1.418 \pm 1.11 \times 10^{-4}$ & 0.030 \\
\hline GO:0006281 & DNA repair & $0.473 \pm 6.38 \times 10^{-5}$ & $0.494 \pm 4.73 \times 10^{-5}$ & 0.030 \\
\hline GO:0046872 & metal ion binding & $0.438 \pm 1.31 \times 10^{-4}$ & $0.472 \pm 3.83 \times 10^{-5}$ & 0.040 \\
\hline GO:0005694 & chromosome & $0.400 \pm 7.23 \times 10^{-5}$ & $0.423 \pm 5.30 \times 10^{-5}$ & 0.030 \\
\hline GO:0006265 & DNA topological change & $0.368 \pm 6.21 \times 10^{-5}$ & $0.392 \pm 5.16 \times 10^{-5}$ & 0.020 \\
\hline GO:0006520 & cellular amino acid metabolic process & $0.340 \pm 1.24 \times 10^{-4}$ & $0.384 \pm 1.06 \times 10^{-4}$ & 0.030 \\
\hline GO:0006313 & transposition, DNA-mediated & $0.332 \pm 2.62 \times 10^{-4}$ & $0.253 \pm 1.33 \times 10^{-4}$ & 0.030 \\
\hline GO:0004803 & transposase activity & $0.329 \pm 2.58 \times 10^{-4}$ & $0.251 \pm 1.32 \times 10^{-4}$ & 0.030 \\
\hline GO:0044237 & cellular metabolic process & $0.228 \pm 9.23 \times 10^{-5}$ & $0.202 \pm 4.39 \times 10^{-5}$ & 0.040 \\
\hline GO:0015986 & ATP synthesis coupled proton transport & $0.204 \pm 2.50 \times 10^{-5}$ & $0.216 \pm 4.15 \times 10^{-5}$ & 0.040 \\
\hline GO:0008033 & tRNA processing & $0.194 \pm 4.29 \times 10^{-5}$ & $0.223 \pm 6.32 \times 10^{-5}$ & 0.010 \\
\hline GO:0003918 & $\begin{array}{l}\text { DNA topoisomerase (ATP-hydrolyzing) } \\
\text { activity }\end{array}$ & $0.170 \pm 4.77 \times 10^{-5}$ & $0.191 \pm 4.02 \times 10^{-5}$ & 0.010 \\
\hline GO:0008652 & $\begin{array}{l}\text { cellular amino acid biosynthetic process } \\
\text { transferase activity, ( alkyl or aryl }\end{array}$ & $0.162 \pm 2.82 \times 10^{-5}$ & $0.176 \pm 4.13 \times 10^{-5}$ & 0.030 \\
\hline GO:0016765 & groups) & $0.140 \pm 8.36 \times 10^{-5}$ & $0.163 \pm 3.63 \times 10^{-5}$ & 0.030 \\
\hline GO:0016820 & hydrolase activity ( acid anhydrides) & $0.129 \pm 3.20 \times 10^{-5}$ & $0.151 \pm 5.99 \times 10^{-5}$ & 0.010 \\
\hline GO:0016868 & $\begin{array}{l}\text { intramolecular transferase activity, } \\
\text { phosphotransferases }\end{array}$ & $0.128 \pm 2.99 \times 10^{-5}$ & $0.144 \pm 4.11 \times 10^{-5}$ & 0.020 \\
\hline GO:0016740 & transferase activity (methyl) & $0.117 \pm 5.69 \times 10^{-5}$ & $0.101 \pm 2.52 \times 10^{-5}$ & 0.030 \\
\hline GO:0006754 & ATP biosynthetic process & $0.112 \pm 3.40 \times 10^{-5}$ & $0.124 \pm 3.51 \times 10^{-5}$ & 0.040 \\
\hline GO:0003964 & RNA-directed DNA polymerase activity & $0.110 \pm 4.97 \times 10^{-5}$ & $0.073 \pm 4.49 \times 10^{-5}$ & 0.000 \\
\hline GO:0006278 & RNA-dependent DNA replication & $0.110 \pm 4.97 \times 10^{-5}$ & $0.073 \pm 4.49 \times 10^{-5}$ & 0.000 \\
\hline GO:0005506 & iron ion binding & $0.104 \pm 2.08 \times 10^{-5}$ & $0.115 \pm 1.29 \times 10^{-5}$ & 0.000 \\
\hline GO:0000105 & histidine biosynthetic process & $0.104 \pm 7.35 \times 10^{-5}$ & $0.125 \pm 3.75 \times 10^{-5}$ & 0.030 \\
\hline GO:0045454 & cell redox homeostasis & $0.087 \pm 5.74 \times 10^{-5}$ & $0.071 \pm 3.37 \times 10^{-5}$ & 0.040 \\
\hline GO:0008237 & metallopeptidase activity & $0.083 \pm 1.79 \times 10^{-5}$ & $0.074 \pm 2.03 \times 10^{-5}$ & 0.020 \\
\hline GO:0008616 & queuosine biosynthetic process & $0.080 \pm 2.85 \times 10^{-5}$ & $0.072 \pm 2.09 \times 10^{-5}$ & 0.050 \\
\hline
\end{tabular}


Several significant changes in protein families associated with cellular metabolism and energy production were identified. The LWB-enriched diet resulted in a significant change of GO terms associated with cellular REDOX homeostasis (GO:0045454), iron ion binding (GO:0005506), and metal ion binding (GO:0046872) (Table 2). Gene Ontology analysis identified a higher abundance of $0.02 \%$ in regards to proteins associated with akyl and aryl transferase (GO:0016765), acid anhydride hydrolase (GO:0016820), and a phosphotransferase (GO:0016868) in the proximal colon microbiota of rat fed a LWB-enriched diet. Conversely, the abundance of proteins associated with methyl transferase activity (GO:0016740) and metaollopeptidase activity (GO:0008237) was significantly reduced by the LWB diet. In addition, GO terms associated with ATP biosynthetic processes and ATP synthesis couple to proton transport were also significantly increased as a result of dietary enrichment of LWB (Table 2). A significant increase in 2,4-dienoyl-CoAreductase (K00219) and a decrease in galacturan 1,4- $\alpha$-galacturonidase (K01213) was observed as a result of the LWB diet (Figure 2).

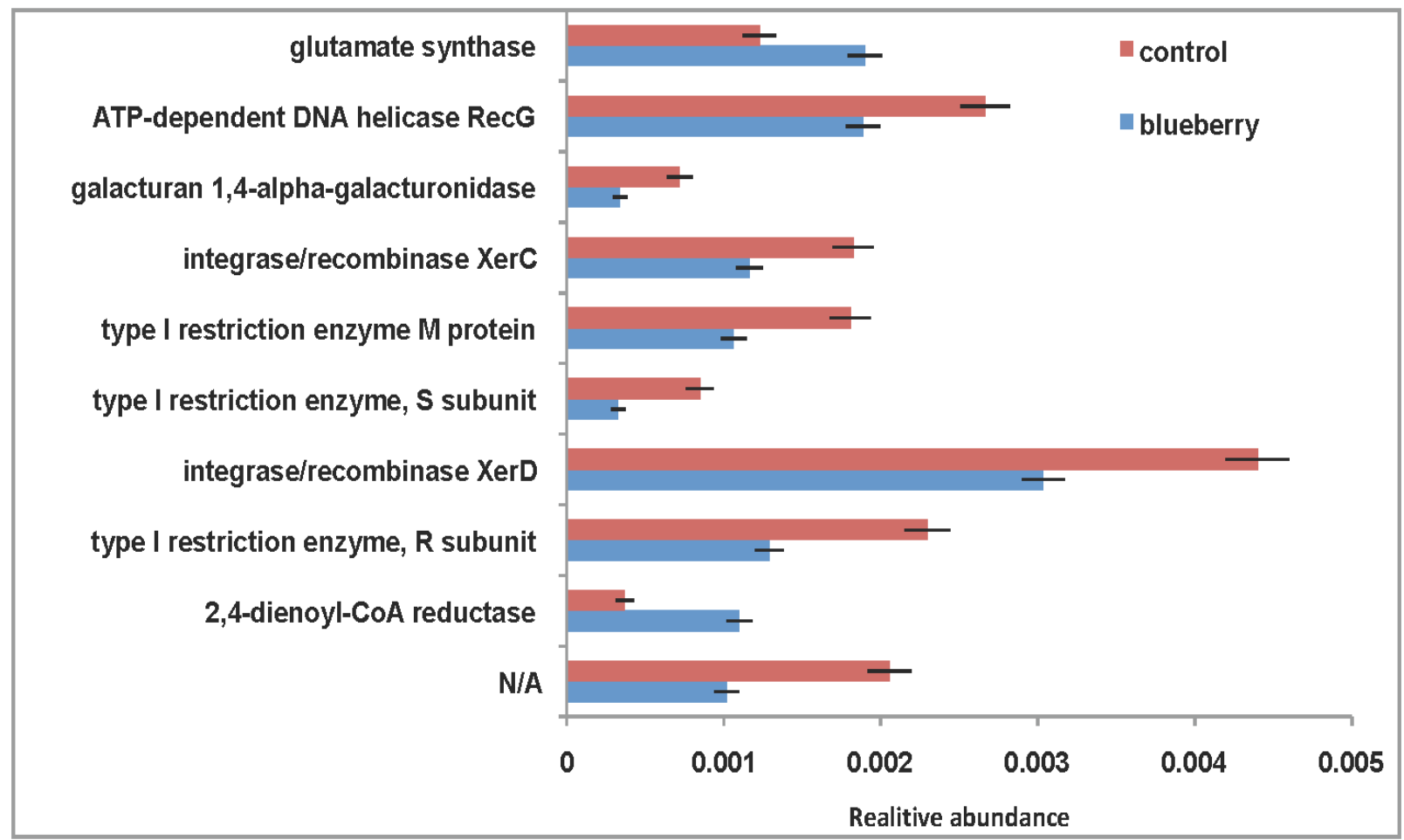

Figure 2: KEGG pathways of the rat proximal colon microbiota impacted $(\mathrm{P}<0.05$ and false discovery rate $(\mathrm{FDR})<10 \%)$ by LWB-enriched diet.

Several significant changes were observed with respect to DNA transcription, modification, recombination, and repair. The annotation used Pfam protein family database also indicated that the LWB diet enhance bacterial regulatory proteins associated with DNA topoisomerase and the nucleoid partitioning protein ParB (Table 3). The LWB observed a reduction the relative abundance of Pfam proteins involved with reverse transcriptase and transcription repressors (TetR family) (Table 3). As Table 2 indicates, the relative abundance of proteins annotated to GO terms associated with DNA repair, topological change, topoisomerase, and chromosome 
maintenance was significantly increased by the LWB diet. In contrast, transposition, transposase, and reverse transcriptase were observed at a lower abundance in the LWB diet (Table 2). Evidence of lower transposition activity in LWB diet can be seen in the decreased abundance of KEGG proteins related to type I restriction enzymes ( $\mathrm{R}$ subunit; $\mathrm{S}$ subunit; and $\mathrm{M}$ protein), RecG, and integrase/recombinase (XerD 0.14 and XerC) (Figure 2).

Table 3. Pfam protein families significantly impacted in the proximal colon microbiota of rats fed a lowbush wild blueberry enriched diet.

\begin{tabular}{|c|c|c|c|c|}
\hline Pfam_ID & Pfam Description & Control & Blueberry & *P value \\
\hline PF00165 & $\begin{array}{l}\text { Bacterial regulatory helix-turn-helix } \\
\text { proteins, AraC }\end{array}$ & $0.491 \pm 4.00 \times 10^{-4}$ & $0.371 \pm 3.34 \times 10^{4}$ & 0.050 \\
\hline PF00496 & $\begin{array}{l}\text { Bacterial extracellular solute-binding } \\
\text { proteins, family } 5\end{array}$ & $0.113 \pm 1.05 \times 10^{-4}$ & $0.164 \pm 1.88 \times 10^{-4}$ & 0.040 \\
\hline PF07993 & Male sterility protein & $0.121 \pm 8.35 \times 10^{-5}$ & $0.095 \pm 7.12 \times 10^{-5}$ & 0.040 \\
\hline PF01073 & $\begin{array}{l}\text { 3-beta hydroxysteroid } \\
\text { dehydrogenase/isomerase family }\end{array}$ & $0.135 \pm 9.10 \times 10^{-5}$ & $0.106 \pm 7.56 \times 10^{-5}$ & 0.040 \\
\hline PF01943 & Polysaccharide biosynthesis protein & $0.112 \pm 7.98 \times 10^{-5}$ & $0.089 \pm 4.30 \times 10^{-5}$ & 0.030 \\
\hline PF01551 & Peptidase family M23 & $0.138 \pm 2.95 \times 10^{-5}$ & $0.148 \pm 2.95 \times 10^{-5}$ & 0.030 \\
\hline PF09820 & Predicted AAA-ATPase & $0.126 \pm 1.06 \times 10^{-4}$ & $0.079 \pm 1.42 \times 10^{-4}$ & 0.030 \\
\hline PF00266 & Aminotransferase class- $\mathrm{V}$ & $0.248 \pm 8.40 \times 10^{-5}$ & $0.276 \pm 5.66 \times 10^{-5}$ & 0.030 \\
\hline PF00275 & $\begin{array}{l}\text { 5-enolpyruvylshikimate-3-phosphate } \\
\text { synthase }\end{array}$ & $0.139 \pm 1.21 \times 10^{-4}$ & $0.179 \pm 8.58 \times 10^{-5}$ & 0.030 \\
\hline PF02195 & ParB-like nuclease domain & $0.116 \pm 5.42 \times 10^{-5}$ & $0.138 \pm 5.54 \times 10^{-5}$ & 0.020 \\
\hline PF00497 & $\begin{array}{l}\text { Bacterial extracellular solute-binding } \\
\text { proteins, family } 3\end{array}$ & $0.119 \pm 2.51 \times 10^{-5}$ & $0.107 \pm 3.40 \times 10^{-5}$ & 0.020 \\
\hline PF00083 & Sugar (and other) transporters & $0.114 \pm 1.13 \times 10^{-4}$ & $0.079 \pm 5.24 \times 10^{-5}$ & 0.020 \\
\hline PF00440 & $\begin{array}{l}\text { Bacterial regulatory proteins, tetR } \\
\text { family }\end{array}$ & $0.130 \pm 8.76 \times 10^{-5}$ & $0.098 \pm 7.08 \times 10^{-5}$ & 0.020 \\
\hline PF00395 & S-layer homology domain & $0.331 \pm 9.70 \times 10^{-4}$ & $0.661 \pm 5.14 \times 10^{-4}$ & 0.020 \\
\hline PF00521 & $\begin{array}{l}\text { DNA gyrase/topoisomerase IV, } \\
\text { subunit A }\end{array}$ & $0.120 \pm 4.28 \times 10^{-5}$ & $0.145 \pm 7.08 \times 10^{-5}$ & 0.020 \\
\hline PF00078 & $\begin{array}{l}\text { Reverse transcriptase } \\
\text { dependent DNA polymerase) }\end{array}$ & $0.139 \pm 1.01 \times 10^{-4}$ & $0.102 \pm 6.59 \times 10^{-5}$ & 0.010 \\
\hline PF00294 & 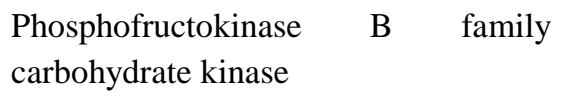 & $0.183 \pm 8.04 \times 10^{-5}$ & $0.212 \pm 4.34 \times 10^{-5}$ & 0.010 \\
\hline PF02653 & $\begin{array}{l}\text { Branched-chain amino acid transport } \\
\text { system }\end{array}$ & $0.153 \pm 1.49 \times 10^{-4}$ & $0.243 \pm 2.13 \times 10^{-5}$ & 0.010 \\
\hline PF05738 & Cna protein B-type domain & $0.226 \pm 1.34 \times 10^{-4}$ & $0.356 \pm 2.11 \times 10^{-5}$ & 0.001 \\
\hline PF08238 & Sel1 repeat & $0.182 \pm 1.57 \times 10^{-4}$ & $0.343 \pm 2.59 \times 10^{-4}$ & 0.001 \\
\hline
\end{tabular}

\section{DISCUSSION:}

Bio-protective effects of polyphenols and other nutrients observed in vitro are not completely transferable to health effects observed in vivo; therefore, it is important to use in vivo models to understand how they are metabolized $[11,12,16,18-20]$. It is imperative to investigate the biological intermediaries in nutrient digestion to gain insight in how bio-protective affect can be 
transferred to the host. Previous studies demonstrated that diet could impact the metabolic potential of the core gut microbiota by influencing energy harvest, metabolic disorders, and immunity $[2,21,22]$. In this study, we present evidence that a LWB-enriched diet may impact gut metabolic activities, REDOX equilibrium, and DNA maintenance mechanisms. Our results provide insight into microbial community's response to blueberries and should help to elucidate the potential benefits in the gut and host.

The energy rich environment of the proximal colon selects for bacteria that are highly competitive and can accommodate a "feast or famine" lifestyle [23]. Bacteria that reside in the gut must be able to sequester nutrients in order to replicate quickly and avoid damage from intestinal enzymes and bile salts [23]. In the present study, genomic data from the core microbiota reveals a high degree of genes associated with macromolecule transport and metabolism of complex carbohydrates. All the databases utilized in this experiment demonstrated similar protein families in high percent abundance, namely, ABC transport systems, followed by histidine metabolism and $\beta$-galactosidase. The results did not detect any significant changes in overall sugar metabolism (Figure 1) or $\beta$-glucosidase or $\beta$-glucuronidase abundance amongst diets. However, results did reflect a decrease in the abundance of polysaccharide biosynthetic protein and sugar transporters, and 1,4 $\alpha$-galacturonidase in the LWB-enriched diet group, and an increase in phosphofructokinase. The data from the COG class observed significant changes in amino acid metabolism. A higher abundance of protein families associated with cellular amino acid biosynthetic and metabolic processes was detected in the LWB-enriched diet group. Like most cells, bacterial amino acid metabolism is in delicate equilibrium with sugar utilization, since many of the substrates are shared. This study demonstrates a possible shift in this equilibrium influenced by the substitution of dextrose with LWB. Over all, these functional profiles coincide with the ones indentified in digestive tract of microbiota isolated from higher mammals [17]. This is important to note, because therapeutic dietary interventions should not affect the global regulation of metabolic genes, rather target specific genes involved with cellular function.

Research linking LWB with gastrointestinal health demonstrated shifts in the microbial community after dietary treatment [7, 11, 18]. Previous work has observed the enrichment of Bifidobacteria in humans fed LWB in a double blind crossover study [11]. The shifts toward increased biodiversity are a likely reflection of the diverse way that microbes catabolized the diverse LWB compounds, especially polyophenol, and the capability for anaerobic digestion in gastrointestinal environments [23]. The enrichment of LWB in the diet may change certain pathways that the gut microbiota uses, possibly modulating the potential for energy harvest. Previous studies demonstrate that anthocyanidin glycosides are hydrolyzed extensively by the intestinal microflora [7, 24]. The present study detected higher levels of tranferases and hydrolyases associated with alkyl, aryl, and anhydride groups that may be important in catabolizing polyphenols. An increased abundance in central metabolism enzymes 2,4-dienoylCoA (DCA) reductase was also observed in the LWB enriched diet (figure 2). In most microorganisms, DCA reductase is an enzyme typically associated with the reduction of conjugated cis-double bonds in $\beta$-oxidation of fatty acids. The bacterium Thauera aromatica uses 1,5-dienoyl-CoA oxidoreductase (DCO) as electron donor for the reduction of aromatic compounds to benzoyl-CoA, a common intermediate of both the anaerobic and aerobic benzoate 
degradation pathways [25]. It is possible that the byproducts of polyphenols catabolism can enter metabolic pathways and potentiate an additional energy source for the core microbiota.

Chromosome maintenance for bacteria is important for surviving and adapting to the highly competitive environment. The present study revealed that the LWB-enriched diet induced a higher abundance of proteins associated with DNA repair, topological change, topoisomerase, and chromosome maintenance, while having a lower abundance of proteins associated with reverse transcriptase activity, as well as, transferase and transpoase activity (Table 2). In addition, the relative abundance of type I restriction enzyme ( $R$ subunit, $S$ subunit, and $M$ protein), recombinase (RecG), and integrase/recombinase (XerD and XerC) was decreased in the LWB diet (Figure 2). The results in the present study reflect lower potential for horizontal integration of plasmids and lower phage/transposon activity in the LWB diet, suggesting that the genome at the time of sampling was more stable. Bacteria in the gut are outnumbered ten to one by phage, most of which are non-lytic, playing an important role in transferring genes [26]. Previous studies have demonstrated the ability of bioflavanoids to inhibit viral retro transcriptase in murine cells, and their ability to bind the viral protein coat inhibiting their function.

The activity of phages may indicate the state of the gut community health and the role of LWB in this delicate balance needs to further exploration.

Nutrients derived from LWB or their metabolites may have protective effects on microorganisms. Compounds isolated from LWB have demonstrated antioxidant properties and have demonstrated their impact upon DNA modification, maintenance, and repair [27]. Cell culture studies cells demonstrated that treatment of anthocyanins had positive effects on elevating the antioxidant capacity, including activated expression of glutathione-related enzymes: glutathione reductase, glutathione peroxidase, and glutathione $S$-transferase [28]. In addition, animal feeding studies with LWB-enriched diets demonstrate a $35 \%$ decrease in DNA damage in the blood plasma, suggesting a potential reduction in damage incurred by peroxides (Del Bo et al. 2010). The present study detected lower levels of protein families associated with REDOX homeostasis in conjunction with higher levels of metal binding proteins in the proximal colon microbiota of rats fed a LWB-enriched diet. Oxidative stress may also decrease the sequestration of iron by bacteria, increasing the pools of reactive iron in the cell potentiating the harm caused by Fenton chemistry [29]. Manganese, in particular, has been increasingly appreciated as a central player in the resistance to ROS, and the Mn:Fe ratio within cells has emerged as a key parameter for protecting proteins against radical mediated damage [29]. Blueberries, in particular, are noted for their high manganese content [30]. The sequestration of iron and other metals ion may reduce the burden on enzymes responsible for diffusing oxygen radicals.

\section{CONCLUSIONS:}

With efforts toward personalized medicine well under way, understanding the gut microbiota functional metabolic capacity is a necessary measure in the design of individualized treatment. Understanding nutrient microbiome interactions will help to substantiate health claims about LWBs. However, more is needed to unravel the in situ exposure mechanisms and elucidate their effects on the host. The present study demonstrates lowbush wild blueberries may have influence on nutrient utilization, oxidative stress, and DNA maintenance in the gut, and could 
affect the availability bio-protective compounds. Although the direct link between the gut microbial function and polyphenols perceived health effects has not been concretely established, this information may be used in a clinical setting to design diet interventions targeting oxidative stress pathways.

Authors' Contributions: Authors contributed to this article equally.

Acknowledgements: This research was supported by Wild Blueberry Commission of Maine, USDA National Needs Graduate Fellowship Competitive (award \#:2007-38420-17764), UMaine Center for Excellence in Teaching and Assessment Active Student Learning Micro-Grants, and the Maine Agricultural and Forest Experiment Station at the University of Maine with external publication number 3250 .

Abbreviations: Lowbush wild blueberry (LWB), Sprague Dawley (SD), Clusters of Orthologous Groups (COG), Kyoto Encylopedia of Genes and Genomes (KEGG), Protein Families (Pfam), Whole Genome Sequencing (WGS), Gene Ontology (GO).

\section{REFERENCE:}

1. Possemiers S, Bolca S, Verstraete W, Heyerick A: The intestinal microbiome: A separate organ inside the body with the metabolic potential to influence the bioactivity of botanicals. Fitoterapia 2011, 82(1):53-66.

2. Muegge BD, Kuczynski J, Knights D, Clemente JC, Gonzalez A, Fontana L, Henrissat B, Knight R, Gordon JI: Diet drives convergence in gut microbiome functions across mammalian phylogeny and within humans. Science 2011, 332(6032):970-974.

3. Wolfe KL, Liu RH: Structure-activity relationships of flavonoids in the cellular antioxidant activity assay. J. Agri. Food Chem 2008, 56(18):8404-8411.

4. Wu XL, Beecher GR, Holden JM, Haytowitz DB, Gebhardt SE, Prior RL: Concentrations of anthocyanins in common foods in the United States and estimation of normal consumption. J. Agri. Food Chem. 2006, 54(11):4069-4075.

5. Joseph JA, Fisher DR, Bielinski D: Blueberry extract alters oxidative stress-mediated signaling in COS-7 cells transfected with selectively vulnerable muscarinic receptor subtypes. J. Alzheimers Dis. 2006, 9(1):35-42.

6. Lacombe A, Wu VCH, White J, Tadepalli S, Andre EE: The antimicrobial properties of the lowbush blueberry (Vaccinium angustifolium) fractional components against foodborne pathogens and the conservation of probiotic Lactobacillus rhamnosus. Food Microbiol. 2012, 30(1):124-131.

7. Molan AL, Lila MA, Mawson J, De S: In vitro and in vivo evaluation of the prebiotic activity of water-soluble blueberry extracts. World J. Microbiol. Biotechnol 2009, 25(7):1243-1249.

8. Kahle K, Kraus M, Scheppach W, Ackermann M, Ridder F, Richling E: Studies on apple and blueberry fruit constituents: Do the polyphenols reach the colon after ingestion? Mol. Nut. Food Res. 2006, 50(4-5):418-423.

9. van Duynhoven J, Vaughan EE, Jacobs DM, Kemperman RA, van Velzen EJJ, Gross G, Roger LC, Possemiers S, Smilde AK, Dore J et al: Metabolic fate of polyphenols in the human superorganism. PNAS. 2011, 108:4531-4538. 
10. Serafini M, Peluso I, Raguzzini A: Session 1: Antioxidants and the immune system flavonoids as anti-inflammatory agents. Proc. Nutr. Soc. 2010, 69(3):273-278.

11. Vendrame S, Guglielmetti S, Riso P, Arioli S, Klimis-Zacas D, Porrini M: Six-week consumption of a wild blueberry powder drink increases Bifidobacteria in the human gut. J. Agri. Food Chem. 2011, 59(24):12815-12820.

12. Kemperman RA, Bolca S, Roger LC, Vaughan EE: Novel approaches for analysing gut microbes and dietary polyphenols: challenges and opportunities. Microbiol. Sgm. 2010, 156:3224-3231.

13. Monagas M, Urpi-Sarda M, Sanchez-Patan F, Llorach R, Garrido I, Gomez-Cordoves C, Andres-Lacueva C, Bartolome B: Insights into the metabolism and microbial biotransformation of dietary flavan-3-ols and the bioactivity of their metabolites. Food \& Function 2010, 1(3):233-253.

14. Turnbaugh PJ, Ley RE, Mahowald MA, Magrini V, Mardis ER, Gordon JI: An obesityassociated gut microbiome with increased capacity for energy harvest. Nature 2006, 444(7122):1027-1031.

15. Reeves PG: Components of the AIN-93 diets as improvements in the AIN-76A diet. J. Nutrition 1997, 127:S838-S841.

16. Del Bo C, Ciappellano S, Klimis-Zacas D, Martini D, Gardana C, Riso P, Porrini M: Anthocyanin absorption, metabolism, and distribution from a wild blueberry-enriched diet (Vaccinium angustifolium) is affected by diet duration in the Sprague-Dawley rat. J. Agri. Food Chem. 2010, 58(4):2491-2497.

17. Li RW, Wu S, Li W, Huang Y, Gasbarre LC: Metagenome plasticity of the bovine abomasal microbiota in immune animals in response to Ostertagia ostertagi infection. PLoS One. 2011, 6(9).

18. Molan AL, Liu ZJ, Kruger M: The ability of blackcurrant extracts to positively modulate key markers of gastrointestinal function in rats. World $\mathrm{J}$ Microbiol Biotechnol. 2010, 26(10):1735-1743.

19. van Dorsten FA, Grun CH, van Velzen EJJ, Jacobs DM, Draijer R, van Duynhoven JPM: The metabolic fate of red wine and grape juice polyphenols in humans assessed by metabolomics. Mol. Nut. Food Res. 2010, 54(7):897-908.

20. van Duynhoven J, van Velzen E, Gross G, van Dorsten F, Jacobs D, Bingham M, Draijer R, Mulder T, Koning T, Vaughan E et al: NMR-based metalbonomics approches for the asssestment of the metabolic impact od dietary polyphenol on human; 2009. PNAS. 108: 4531-4538.

21. Cani PD, Delzenne NM: The gut microbiome as therapeutic target. Pharmacol Ther. 2011, 130(2):202-212.

22. Diamant MDM, Blaak EE, de Vos WM: Do nutrient-gut-microbiota interactions play a role in human obesity, insulin resistance and type 2 diabetes? Obes Rev. 2011, 12(4):272-281.

23. Vieira-Silva S, Rocha EPC: The Systemic Imprint of Growth and Its Uses in Ecological (Meta) Genomics. Plos Genetics 2010, 6(1):15.

24. Selma MV, Espin JC, Tomas-Barberan FA: Interaction between Phenolics and Gut Microbiota: Role in Human Health. J. Agri. Food Chem. 2009, 57(15):6485-6501. 
25. Thiele B, Rieder O, Jehmlich N, von Bergen M, Mueller M, Boll M: Aromatizing cyclohexa1,5-diene-1-carbonyl-coenzyme a oxidase - Characterization and its role in anaerobic aromatic metabolism. Journal of Biological Chemistry 2008, 283(30):20713-20721.

26. Reyes A, Haynes M, Hanson N, Angly FE, Heath AC, Rohwer F, Gordon JI: Viruses in the faecal microbiota of monozygotic twins and their mothers. Nature 2010, 466(7304):334U381.

27. Del Bo C, Martini D, Vendrame S, Riso P, Ciappellano S, Klimis-Zacas D, Porrini M: Improvement of lymphocyte resistance against $\mathrm{H}(2) \mathrm{O}(2)$-induced DNA damage in SpragueDawley rats after eight weeks of a wild blueberry (Vaccinium angustifolium)-enriched diet. Mutat. Res-Gen. Tox. En. 2010, 703(2):158-162.

28. Shih PH, Yeh CT, Yen GC: Anthocyanins induce the activation of phase II enzymes through the antioxidant response element pathway against oxidative stress-induced apoptosis. J. Agri. Food Chem. 2007, 55(23):9427-9435.

29. Faulkner MJ, Helmann JD: Peroxide Stress Elicits Adaptive Changes in Bacterial Metal Ion Homeostasis. Antioxid. Redox Sign. 2011, 15(1):175-189.

30. Rupasinghe HPV, Clegg S: Total antioxidant capacity, total phenolic content, mineral elements, and histamine concentrations in wines of different fruit sources. J. Food Compos. Anal. 2007, 20(2):133-137. 